\title{
Reaction of Subcutaneous Connective Tissue of Experimental Animals on Bone Marrow Mesenchymal Stromal Cell Coated Hydroxyapatite
}

\author{
Aleksejs Zavorins*, Mara Pilmane*, Girts Salms**, Andrejs Skagers**, Inese Cakstina***, Janis Locs**** \\ *Institute of Anatomy and Anthropology, Riga Stradins University, Latvia \\ **Institute of Stomatology, Riga Stradins University, Latvia \\ *** Laboratory of Biodosimetry and Bioanalistical Methods, University of Latvia, Latvia ****Riga Biomaterials Innovation and \\ Development Center, Riga Technical University, Latvia
}

\begin{abstract}
Summary
Introduction. Hydroxyapatite (HAp) scaffolds have become an alternative to autologous bone grafts in orthopedic surgery, because it is possible to fill larger scale bone defects and of the decreased operation time and complication risks at the graft donor site, a promising method in bone regeneration is to load bone marrow - mesenchymal stromal cells (BM-MSCs) on to a HAp scaffold.

Aim of the Study. The aim of our study is to compare subcutaneous tissue reaction of experimental animals to implantation of HAp and HAp that is coated with BM-MSCs.

Materials and Methods. Autologous BM-MSCs were cultivated from crista iliaca dextra biopsy, 4 HAp ceramic implants were coated with BM-MSC and implanted in subcutaneous tissue on one side and non-coated HAp implants on the other side of the spine of 4 rabbits. Immunohistochemical staining of BM-MSC and connective tissue included TUNEL assay, NFkBp105, HSP 70k, Wnt1, TNF- $\alpha$, VEGF, MMP-2 antibodies, microscoped at 400X magnification and analyzed semiquantitatively.

Results. Routine staining with haemotoxilin and eosin demonstrated formation of granulation tissue around the HAp implant, which was more distinct on the control side. There was an increased number of NFkB p105 positive cells (fibroblasts, myocytes, endotheliocytes) on the experimental side (+++), in comparison to the control side (++). HSP70 and apoptosis index was decreased on the experimental side (++ and $60 \%$ ), in comparison to the control side (+++ and $70 \%$ ). Number of MMP2 positive structures was increased on the control side in the fibrous capsule (++), in comparison to the experimental side, where the result varied $(0-+)$ Conclusions. Cultured BM-MSC activity is partly limited due to the apoptosis, which is probably induced by environmental factors. BM-MSC coated HAp implant stimulates secretion of proliferation marker NFkBp105 in subcutaneous tissue, decreases cell stress, apoptosis and tissue degradation (decreased HSP 70, apoptotic cell count and MMP 2) to improve the local tissue quality and proving BM-MSC tissue protective effect.
\end{abstract}

Key words: bone marrow mesenchymal stromal cells, hydroxyapatite, nuclear factor kappa-B

\section{INTRODUCTION}

Hydroxyapatite (HAp) scaffolds have become an alternative to autologous bone grafts in orthopedic surgery, because of the decreased operation time and complication risks at the graft donor site (e.g.crista iliaca), such as infection, excessive traumatization and postoperative pain (10). Up to $39 \%$ of patients complain of chronic pain at the donor site, other complaints include nerve damage, disturbed gait, hernias and fractures. HAp scaffolds have an advantage over autologous grafts, because it is possible to fill larger scale bone defects with it due to the limited amounts of available autologous graft material (7).

Principal features of biomaterials that could substitute bone grafts, including osteoconductivity, osteoinductivity and osteogenecity, were postulated by Marshall Urist in 1965 on the basis of demineralized bone matrix (DBM) (10). Osteoconductivity describes two processes: osteoconversion (the ability of bone tissue to grow into the scaffold and replace it) and osteointegration (the ability of the scaffold to fixate to surrounding bone tissue) (8). HAp is a highly crystalline form of calcium phosphate and is similar to the mineralized phase of the bone. It has a porous structure, which defines the excellent osteoconductive potential of HAp $(5,12)$. Osteoconduction is a threedimensional process, which includes vascularization (in-growth of capillaries with perivascular tissue and subsequent migration of osteoprogenitor cell. There is evidence that, when comparing to a control group, where no scaffold is used, use of HAp to fill bone defects improves tissue organization, decreases amounts of fibrous scar tissue by promoting ossification, it also stimulates angiogenesis and allows forming blood vessel shunts through the defect (11). Another study stressed osteoconductivity of HAp in vivo in 2.5 year follow-up study on dogs (18). Osteoinductivity describes the ability of this biomaterial to stimulate bone tissue formation through various biochemical pathways with certain growth factors and cytokines $(4,7)$. HAp has a relatively high biocompatibility. In an in vivo study on mice inflammatory reaction (proved histologically and by reverse transcriptase Polymerase Chain Reaction (RT-PCR) using IL-l as an inflammatory marker) to subcutaneous HAp implantation was significantly lower 
than to implantation of DBM (10). Several in vivo studies state the possibility to utilize HAp as carrier of growth factors to induce ossification, including bone morphogenetic protein - 2 (BMP-2) $(5,19)$. BMPs induce bone formation and induce the undifferentiated mesenchymal cells to differentiate to osteogenic cells. Other factors that seem to promote bone formation are insulin-like growth factor (IGF-1), basic fibroblast growth factor (bFGF) and vascular endothelial growth factor (VEGF) $(4,12)$. Osteogenecity is obtained if biomaterial scaffold is coated or by any other means has access to osteogenic precursor cells, which subsequently differentiate and form bone tissue $(7,12)$.

Stems cells are undifferentiated cells that can proliferate and renew its population and have a potential to differentiate into various cell lineages (12). Stem cells in regenerative medicine have been applied to such organs as nerves, blood vessels and heart (17). Mesenchymal stem or stromal cells (MSCs) can be acquired from bone marrow by aspiration with a puncture needle $(13,15$, 17). MSCs account for $0.0015 \%$ of subcellular bone marrow component (17), and have been successfully used for bone regeneration in in vivo studies on animals and humans (14). MSCs can differentiate into different types of cells, including chondrocytes, myocytes, and osteoblasts (15). One of the main issues with exploiting MSCs in regenerative medicine is the inability to provide these cells with adequate biochemical stimuli, needed to guide the differentiation of MSCs into the necessary lineage, and, therefore, several studies point out the need to add an exogenous soluble growth factor $(12,13)$. Another issue is the medium, by which MSCs are delivered into tissue so that these cells are sustained at the necessary site $(12,13)$. A promising method in bone regeneration is to load MSCs on to a HAp scaffold; several studies have demonstrated better results with this method, than when single HAp scaffold was implanted $(13,15,17)$. One in vivo study on dogs compared HAp coated with MSCs with a control group, where HAp was implanted into the bone defect without the coating. The results demonstrated better vascularization in regenerated tissue in the experimental group. Authors also pointed out that HAp in the control group was encapsulated by fibrotic tissue, which was not observed in the experimental group (17).

\section{AIM OF THE STUDY}

The aim of our study is to compare subcutaneous tissue reaction of experimental animals to implantation of HAp and HAp that is coated with BM-MSCs.I

\section{MATERIALS AND METHODS}

Implants:

HAp implants were produced in Riga Technical University Riga Biomaterials Innovation and Development centre. The wet precipitation reaction between calcium hydroxide and phosphoric acid was used to obtain the hydroxyapatite powder. The powder was formed in porous shape and sintered at $1150^{\circ} \mathrm{C}$ for two hours. Experimental animals and BM-MSCs:
The Animal Ethics Committee of Latvian Food and Veterinary Administration gave permission to use rabbits for morphofunctional study (Number 24, 02.07.2010). In the study 4 New Zealand male rabbits were included. Biopsy of spongious bone tissue was taken from crista iliaca dextra (January 26, 2011) of each rabbit, subsequently this material was grinded and incubated with Type XI collagenase. The cells from this substance were than cultured in an environment containing Dulbecco's Modified Eagle's Medium (DMEM), fetal bovine serum 20\% (FBS) and penicillin/ streptomycin. Cells went through three cell passages, achieving at least $70 \%$ confluence of adherent cells. Bone marrow mesenchymal stromal cells (BM-MSCs) were obtained from bone marrow of 4 rabbits. These cells are not proven to be MSCs yet, however they are not haemopoetic cells. Obtained cells were adherent and morphologically similar to MSCs. BM-MSCs were immunohistochemically evaluated in Part I of the experiment that is outlined in the section called Staining and microscopical evaluation (Table 1 ).

On February 9, 2011 HAp implants were coated with 100 '000 BM-MSCs. The autologous BM-MSCs coated HAp implants were implanted into the subcutaneous tissue of the first 2 rabbits on the right side of the spine the experimental side. On the left side of the spine - the control side - single HAp implants without BM-MSCs coating were implanted.

On March 8, 2011 euthanasia by air embolisation was performed. Rabbits received general anaesthesia with Ketamini $15 \mathrm{mg} / \mathrm{kg}$ and Midazolami $0.5 \mathrm{mg} / \mathrm{kg} \mathrm{i} / \mathrm{m}$ and additional local anaesthesia with Sol. Lidocaini $2 \%(4 \mathrm{mg} / \mathrm{kg})$. Afterwards specimens of subcutaneus tissue in the region with HAp implants were obtained and immersed into Stefanini solution. These specimens were immunohistochemically evaluated in Part II of the experiment that is outlined in the section called Staining and microscopical evaluation.

Similarly, on March 8, 2011 HAp implants coated with $100^{\prime} 000$ BM-MSCs and uncoated HAp were implanted on the right and left side of the spine of the remaining 2 rabbits. The specimens for histological analysis were obtained on June 13, 2011. This is outlined in Part III of the experiment.

Staining and microscopical evaluation:

The experiment can be divided into 3 parts. Part I:

Slides containing 20'000 cells were prepared using Lab-tek II Chamber slide. Material was stained immunohistochemically with several antibodies:

Nuclear Factor - кBl pl05 (NFkB pl05/ p50, 1:100 solution, Abcam, UK)

Heat Shock Protein 70 (HSP 70, 1:100, Abcam, UK)

In order to evaluate apoptosis staining with TUNEL kit (Roche, Germany) was also used.

Part II:

Specimens were stained with haemotoxilin and eosin and immunohistochemically with several types of antibodies: NFkB pl05 (NFkB pl05/ p50, 1:100 solution, Abcam, UK), TUNEL kit (Roche, Germany), 
HSP 70 (HSP 70, 1:100, Abcam, UK) and Wntl (1:100 solution, Abcam, UK).

Part III

Specimens were stained with haemotoxilin and eosin and immunohistochemically with several types of antibodies: NFkB pl05 (NFkB pl05/ p50, 1:100 solution, Abcam, UK), TUNEL kit (Roche, Germany), HSP 70 (HSP 70, 1:100, Abcam, UK) and Wntl (1:100 solution, Abcam, UK), tumor necrosis factor- $\alpha$ (TNF- $\alpha$, 1:100 solution, Abcam, UK), fibroblast growth factor receptor 1 (FGFRl, 1:100 solution, Abcam, UK), vascular endothelial growth factor (VEGF, 1:50 solution, Dako, Denmark), matrix-metalloproteinase 2 (MMP-2, 1:100 solution, Leica, UK).

All specimens (3 slides for the experimental side and 1 slide for the control side with each staining) were microscoped and photographed at 200x, 250x, 400x magnification and evaluated semi-quantitively. Depending on the relative quantity of positively stained structures, specimens were rated as having a few positive structures $(+)$, moderate quantity of positive structures $(++)$, numerous quantity of positive structures $(+++)$ and abundant amount of positive structures $(++++)$, and a negative result is equal to 0 . Apoptosis index was also calculated in specimens stained using TUNEL kit.

\section{RESULTS}

In the Part I of the experiment all bone marrow mesenchymal stromal cells have been apoptotic. BMMSCs with ameboid structure in the specimens of the first rabbit demonstrated abundant $(++++)$ expression of NFkB p105 - proliferation and inflammatory marker -, however BM-MSCs with a mesenchymal structure demonstrated a negative result $(0)$ for programmed cell death Table 2, Figure 1. Expression of NFkB pl05 was numerous $(+++)$ in all BM-MSCs in specimens of the second rabbit. Expression of heat shock protein 70 indicator of cell stress - varied in specimens of rabbit 1 from negative $(0)$ in cells with mesenchymal structure to numerous $(+++)$ in cells with amebic structure, and was numerous $(+++)$ in specimens of the second rabbit. In the Part II of the experiment routine staining with haemotoxilin and eosin demonstrated formation of granulation tissue with increased vascularization around the HAp implant, which was more distinct on the control side. There was also inflammatory infiltration on the control side, which was not observed on the experimental side. Apoptosis index (70-90\%), NFkB plo5 (++++), WNTl (++++) and HSP70 (++++) positive structures including endothelial cells, fibroblasts, and leukocytes was similar on both sides.

In the Part III of the experiment, routine microscopy demonstrated formation of new muscle fibers, which was more distinguished on the experimental side, some muscle fibers were vacuolised, though no signs of inflammation have been noticed. On the control side rare peri-vascular inflammation can be noticed, dense fibrous capsule has formed around the HAp implant. There was an increased number of NFkB pl05 positive cells (fibroblasts, myocytes, endotheliocytes) on the experimental side $(+++)$, in comparison to the control side (++) (Figure 2 and 3). HSP70 and apoptosis index was decreased on the experimental side (++ and 60\%), in comparison to the control side (+++ and 70\%) (Figure 4 and 5). Number of MMP2 positive structures was increased on the control side in the fibrous capsule $(++)$, in comparison to the experimental side, where the result varied $(0-+)$.

\section{DISCUSSION}

All cultured bone marrow mesenchymal stromal cells were apoptotic. This indicates that after the BM-MSCs coated implants are implanted into the subcutaneous tissue of the rabbits, these cells will not differentiate further. We speculate that this might be due to the lack of certain cytokines and growth factors, which normally in vivo stimulate osteoblast precursor cells. These factors include bone morphogenetic protein - 2 (BMP2) (6) and IL-3 (1). Several studies also point out the positive role of fibroblast growth factor/ fibroblast growth factor receptor signaling system on the differentiation of osteoprogenitor cells (9). We speculate that these BM-MSCs are capable of inducing proliferation of subcutaneous tissue and regulating inflammatory reactions due to the following reasons.

BM-MSCs in this study stained positive for nuclear factorkappa B p105/ p50. Several recent studies underline the impact of NF-kB signaling pathway on bone formation through both - osteoclasts and osteoblasts. Inactive NF-kB is located in the cells cytoplasm bound to the inhibitor IkB. When activated, IKK (inhibitor of NF- kB kinase) phosphorylates this complex and thus leads to the liberation and transport of NF-kB p50 to the nucleus, where it regulates expression of genes that enhance inflammatory responses and stimulate proliferation. Tumor necrosis factor alpha (TNF- $\alpha$ ) is one of factors that could provoke the NF-kB activation (16). Therefore, it is possible that BM-MSCs may serve as inductors of inflammation and/ or proliferation after BM-MSCs' coated implants are implanted into the subcutaneous tissue.

The NF-kB pathway and its role in regulation of inflammatory response and stimulation of proliferation may explain the lack of distinct fibrous capsule on the experimental side in the III part of our study. The increased levels of NF-kb p 105/p50, decreased apoptosis and tissue degradation on the experimental side may be also connected to the inductive stimuli of the BMMSCs on the HAp implants, even though these cells were apoptotic. This inductive function of BM-MSCs coated HAp implants has been described in several other studies $(3,17)$. There is evidence that BM-MSCs can stimulate angiogenesis by promoting VEGF expression, this on the other hand is beneficial for bone formation (17). Our experiment did not show any distinct difference of VEGF levels on both sides. There is also information that implantation of BM-MSCs has some immunosuppressive effects (3), this may explain the decreased infiltration and decreased formation of fibrous capsule on the experimental side. One study states that BM-MSCs express cytokines that inhibit apoptosis of surrounding cells (2). 


\section{CONCLUSIONS}

1. Cultured BM-MSC activity is partly limited due to the apoptosis, which is probably induced by environmental factors.

2. BM-MSC coated HAp implant stimulates secretion of proliferation marker NFkBpl05 in subcutaneous tissue, decreases cell stress, apoptosis and tissue degradation (decreased HSP 70, apoptotic cell count and MMP 2) to improve the local tissue quality and proving BM-MSC tissue protective effect.

Table 1. Illustrates the algorithm of manipulations held during the course of experiment to obtain specimens of subcutaneous tissue for histological analysis. BM-MSCs coated HAp and uncoated HAp were implanted on the right and left side of the spine in each rabbit.

\begin{tabular}{|c|c|c|c|c|}
\hline & \multicolumn{2}{|c|}{ Part II } & \multicolumn{2}{|c|}{ Part III } \\
\hline & $\begin{array}{c}\text { Rabbit } \\
1\end{array}$ & $\begin{array}{c}\text { Rabbit } \\
2\end{array}$ & $\begin{array}{c}\text { Rabbit } \\
3\end{array}$ & Rabbit 4 \\
\hline $\begin{array}{c}\text { Crista iliaca } d x \\
\text { biopsy }\end{array}$ & \multicolumn{4}{|c|}{ January 26, 2011} \\
\hline $\begin{array}{l}\text { BM-MSC coated } \\
\text { HAp is implanted }\end{array}$ & \multicolumn{2}{|c|}{$\begin{array}{c}\text { February 9, } \\
2011\end{array}$} & \multicolumn{2}{|c|}{ March 8, 2011} \\
\hline $\begin{array}{l}\text { Uncoated HAp is } \\
\text { implanted }\end{array}$ & \multicolumn{2}{|c|}{$\begin{array}{c}\text { February 9, } \\
2011\end{array}$} & \multicolumn{2}{|c|}{ March 8, 2011} \\
\hline $\begin{array}{l}\text { Tissue specimens } \\
\text { are obtained }\end{array}$ & \multicolumn{2}{|c|}{ March 8, 2011} & \multicolumn{2}{|c|}{ June 13, 2011} \\
\hline
\end{tabular}

Table 2. Semiquantitative analysis of immunohistochemical BM-MSCs and subcutaneus tissue staining. + - a few positive structures/ week expression, ++ - moderate quantity of positive structures/ moderate expression, +++ numerous quantity of positive structures/marked expression, ++++ - abundant quantity of positive structures/ abundant expression, and 0 indicates a negative result.

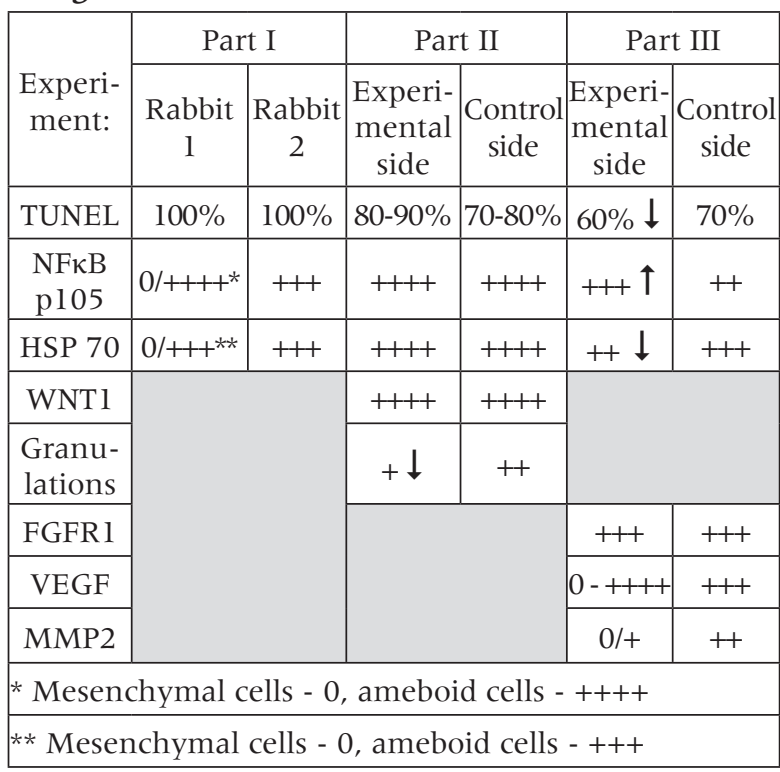

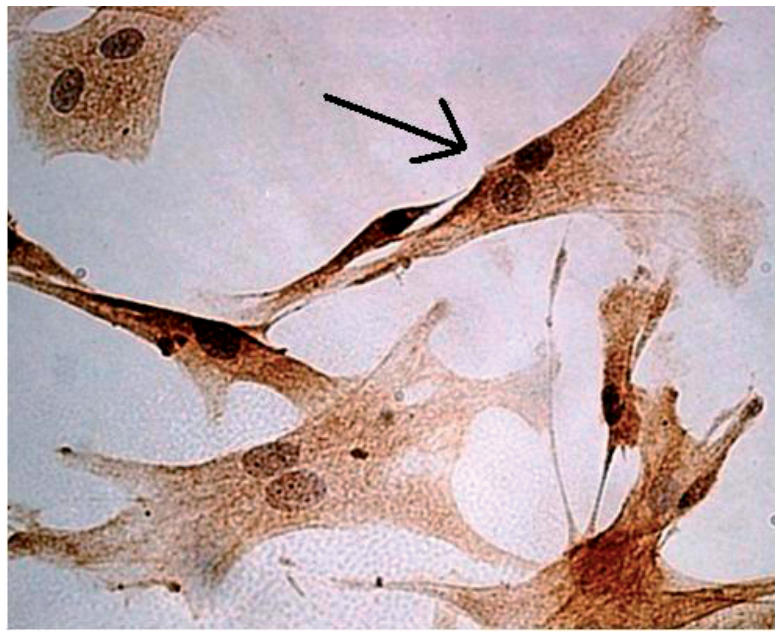

Fig. 1. NF-kB p105 positive bone marrow mesenchymal stromal cells (black arrow), rabbit I. Immunohistochemistry, anti- NF-kB p 105, 400x.

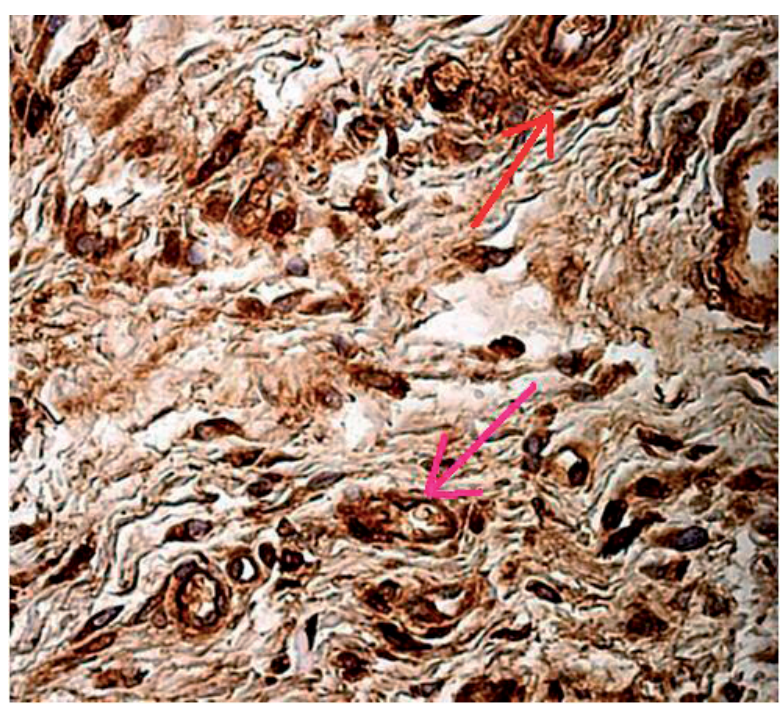

Fig. 2. NF-kB p105 positive endotheliocytes (pink arrow), myocytes (red arrow), experimental side, rabbit II, Immunohistochemistry, anti- NF-kB p105, 400x. 


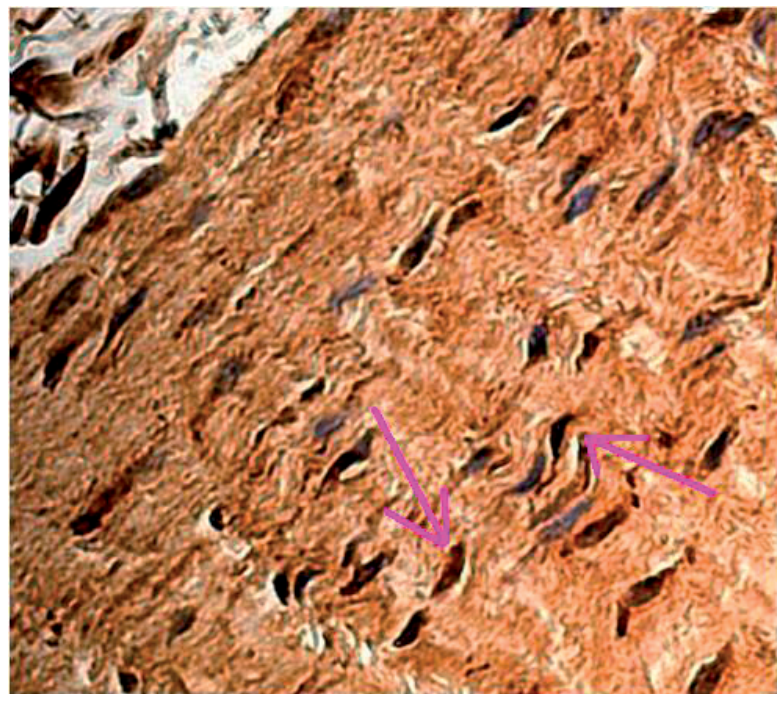

Fig. 3. NF-kB p105 positive fibroblasts in the fibrous capsule (pink arrows), control side, rabbit II. Immunohistochemistry, anti- NF-kB p105, 400x.

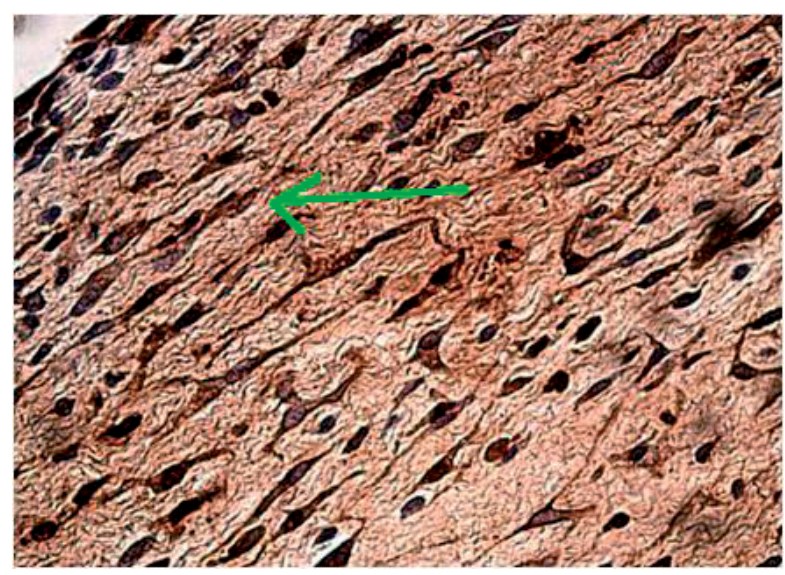

Fig. 4. Apoptosis of fibroblasts in the fibrous capsule (green arrow), control side, rabbit II, TUNEL, 400x.

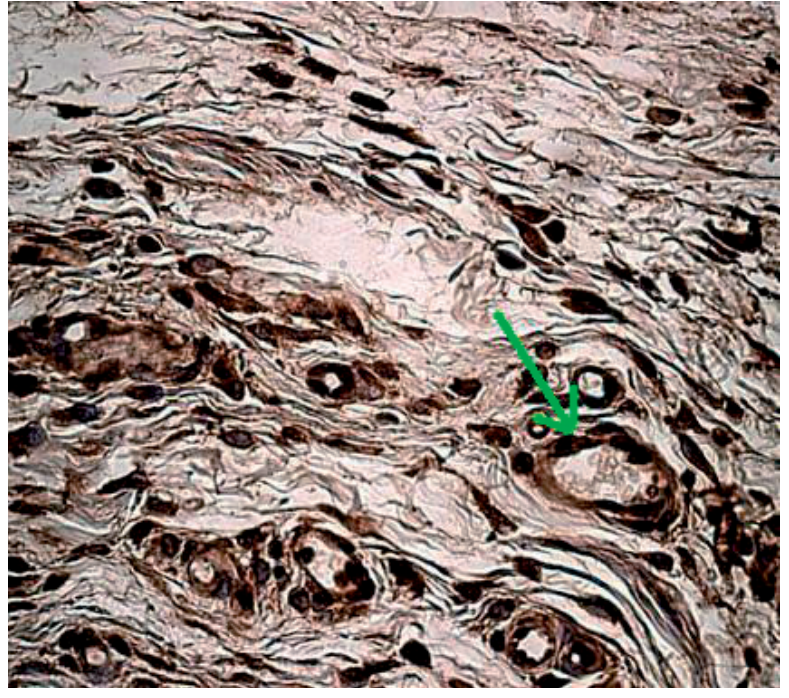

Fig. 5. Apoptosis of endotheliocytes, experimental side (green arrow), rabbit II, TUNEL, 400x

Conflict of interest: None

\section{REFERENCES}

1. Barhanpurkar AP, Gupta N, Srivastava RK et al. IL-3 promotes osteoblast differentiation and bone formation in human mesenchymal stem cells. Biochem Biophys Res Commun. 2012 Feb 24;418(4):669-75. Epub 2012 Jan

2. Boomsma RA, Geenen DL. Mesenchymal stem cells secrete multiple cytokines that promote angiogenesis and have contrasting effects on chemotaxis andapoptosis. PLoS One. 2012;7(4):e35685. Epub 2012 Apr 25.

3. De Miguel MP, Fuentes-Julián S, BlázquezMartínez A et al. Immunosuppressive properties of mesenchymal stem cells: advances and applications. Curr Mol Med. 2012 Apr 18.

4. Ferreira G, Cesari T, Granjeiro J et al. Lack of Repair of Rat Skull Critical Size Defect Treated with Bovine Morphometric Protein Bound to Microgranular Bioabsorbable Hydroxyapatite. Braz Dent J (2004) 15(3): 175-180

5. Fu YC, Nie H, Ho ML et al. Optimized bone regeneration based on sustained release from threedimensional fibrous PLGA/HAp composite scaffolds loaded with BMP-2. Biotechnol Bioeng. 2008 Mar $1 ; 99(4): 996-1006$.

6. Han SH, Kim KH, Han JS et al. Response of osteoblast-like cells cultured on zirconia to bone morphogenetic protein-2. J Periodontal Implant Sci. 2011 Oct;41(5):227-33. Epub 2011 Oct 31.

7. Huber F, Belyaev O, Hillmeier J et al. First histological observations on the incorporation of a novel nanocrystalline hydroxyapatite paste OSTIM® in human cancellous bone BMC Musculoskeletal Disorders 2006, 7:50. 
8. Kokoska M, Friedman C, Castellano R et al. Experimental Facial Augmentation With Hydroxyapatite Cement. Arch Facial Plast Surg. 2004;6:290-294

9. Marie PJ. Fibroblast growth factor signaling controlling bone formation: an update. Gene. 2012 Apr 25;498(1):1-4. Epub 2012 Feb 9.

10. Markel D, Guthrie T, Wu B et al. Characterization of the inflammatory response to four commercial bone graft substitutes using a murine biocompatibility model. Journal of Inflammation Research 2012:5 $13-18$

11. Nandi S, Kundu B, Ghosh S. Efficacy of nanohydroxyapatite prepared by an aqueous solution combustion technique in healing bone defects of goat. J. Vet. Sci. (2008), 9(2), 183-191

12. Nandi S, Roy S, Mukherjee P et al. Orthopaedic applications of bone graft and graft substitutes: a review. Indian J Med Res 132, July 2010, pp 15-30

13. Polini A, Pisignano D, Parodi M et al. Osteoinduction of Human Mesenchymal Stem Cells by Bioactive Composite Scaffolds without Supplemental Osteogenic Growth Factors. PLoS ONE , October 2011, 6(10): e26211.8 pages.

14. Sun-Woong K, Lee JS, Sun Park $M$ et al. Enhancement of In Vivo Bone Regeneration Efficacy of Human Mesenchymal Stem Cells. J. Microbiol. Biotechnol. (2008), 18(5), 975-982

15. Vahabi S; Amirizadeh N, Shokrgozar MA et al. A Comparison between the Efficacy of BioOss, Hydroxyapatite Tricalcium Phosphate and Combination of Mesenchymal Stem Cells in Inducing Bone Regeneration Chang Gung Med J Vol. 35 No. 1 January-February 2012, 8 pages.
16. Yamazaki M, Fukushima H, Shin M et al. Tumor necrosis factor alpha represses bone morphogenetic protein (BMP) signaling by interfering with the DNA binding of Smads through the activation of NFkappaB. J Biol Chem. 2009 Dec 18;284(51):3598795.

17. Yoshioka $\mathrm{M}$, Tanimoto $\mathrm{K}$, Tanne $\mathrm{Y}$ et al. Bone Regeneration in Artificial Jaw Cleft by Use of Carbonated Hydroxyapatite Particles and Mesenchymal Stem Cells Derived from Iliac Bone. International Journal of Dentistry Volume 2012, Article ID 352510, 8 pages

18. Yuan, H., Z. Yang, J. D. de Bruijn, K. de Groot, and $X$. Zhang. Material-dependent bone induction by calcium phosphate ceramics: a 2.5-year study in dog. Biomaterials, 2001. 22:2617-2623

19. Zhang $\mathrm{P}$, Hong $\mathrm{Z}$, Yu $\mathrm{T}$ et al. In vivo mineralization and osteogenesis of nanocomposite scaffold of poly(lactide-co-glycolide) and hydroxyapatite surface-grafted with poly(L-lactide). Biomaterials. 2009 Jan;30(1):58-70.

\section{Address:}

Aleksejs Zavorins,

Anatomijas un antropoloǵijas institūts,

Kronvalda bulv. 9, Rīga, Latvija, LV - 1010

aleksejs.zavorins@gmail.com 